\title{
Beam spin asymmetry benchmark in the spinless meson electroproduction off the scalar target *
}

\section{Chueng-Ryong $\mathbf{J i}^{\dagger}$}

Department of Physics, Box 8202, North Carolina State University, Raleigh, NC 27695-8202

E-mail: crjiancsu.edu

\section{Ho-Meoyng Choi}

Department of Physics, Teachers College, Kyungpook National University, Daegu 41566, Korea

E-mail: homyoung@knu.ac.kr

\section{Andrew Lundeen}

Department of Physics, Box 8202, North Carolina State University, Raleigh, NC 27695-8202

E-mail: ajlundee@ncsu.edu

\section{Bernard L. G. Bakker}

Faculty of Science, Vrije Universiteit, Amsterdam, Netherlands

E-mail: b.1.g.bakker@vu.edu

\begin{abstract}
We discuss the electroproduction of a spinless meson, i.e. pseudoscalar $\left(0^{-+}\right)$or scalar $\left(0^{++}\right)$ meson, off the scalar target. The most general formulation of the differential cross section for the $0^{-+}$or $0^{++}$meson process involves only one or two hadronic form factors, respectively, on a scalar target. The Rosenbluth-type separation of the differential cross section provides the explicit relation between the hadronic form factors and the different parts of the differential cross section in a completely model-independent manner. The absence of the beam spin asymmetry for the pseudoscalar meson production provides a benchmark for the experimental data analysis. The measurement of the beam spin asymmetry for the scalar meson production may also provide a unique opportunity not only to explore the imaginary part of the hadronic amplitude in the general formulation but also to examine the significance of the chiral-odd generalized parton distribution (GPD) contribution in the leading-twist GPD formulation.
\end{abstract}

Light Cone 2019 - QCD on the light cone: from hadrons to heavy ions - LC2019

16-20 September 2019

Ecole Polytechnique, Palaiseau, France

${ }^{*}$ This work was supported in part by the US Department of Energy under Grant No. DE-FG02-03ER41260 and by the National Research Foundation of Korea (NRF) under Grant No. NRF-2017R1D1A1B03033129.

${ }^{\dagger}$ Speaker. 


\section{Salient Features}

Our work investigates exclusive electroproduction of a pseudoscalar $\left(0^{-+}\right)$meson or a scalar $\left(0^{++}\right)$meson off the scalar target [1]. Unlike the case of deeply virtual Compton scattering (DVCS), there is no interference from the Bethe-Heitler process in this investigation. Our work has a major implication for the Beam Spin Asymmetry (BSA) prediction for the $\pi^{0}$ production off the ${ }^{4} \mathrm{He}$ target, which has indeed been measured. The data [2] appear consistent with our benchmark prediction, namely that the BSA should vanish for the pseudoscalar $\left(0^{-+}\right)$meson off the scalar target. We provide a general formulation of hadronic amplitudes in the exclusive electroproduction of a pseudoscalar $\left(0^{-+}\right)$meson or a scalar $\left(0^{++}\right)$meson off the scalar target, which is model-independent and makes no assumptions in regard to the kinematics. We also compare and contrast our findings to those from the leading-twist approach of the generalized parton distributions (GPDs). The result from our analysis implies a significant contribution from the leading-twist chiral-odd GPD $\left(H_{T}\right)$ as it must overcome a $\frac{\sqrt{-t}}{Q}$ suppression factor in order for there to be a non-vanishing BSA in the scalar $\left(0^{++}\right)$meson production off the scalar target. More details of our work can be found in Ref. [1].

\section{General Form of the Cross Section and BSA Calculations}

The total differential cross section can be written in terms of a kinematic factor multiplied with the square amplitude as (see Figure 1 for kinematics definitions)

$$
\mathrm{d} \sigma \equiv \frac{\mathrm{d}^{5} \sigma}{\mathrm{d} y \mathrm{~d} x \mathrm{~d} t \mathrm{~d} \phi_{k^{\prime}} \mathrm{d} \phi_{q^{\prime}}}=\kappa\left\langle|\mathscr{M}|^{2}\right\rangle
$$

where

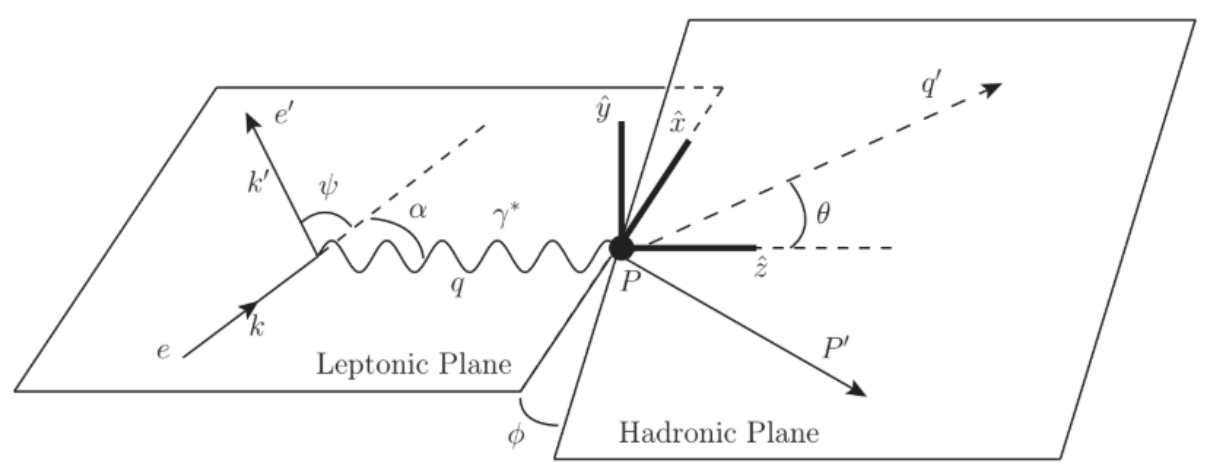

Figure 1: Target Rest Frame (TRF) kinematics. 


$$
\begin{gathered}
\kappa \equiv \frac{1}{(2 \pi)^{5}} \frac{y x}{32 Q^{2} \sqrt{1+\left(\frac{2 M x}{Q}\right)^{2}}} ; q^{2}=-Q^{2} ; \quad y=\frac{P \cdot q}{P \cdot k} ; t=\left(P-P^{\prime}\right)^{2} ; x=\frac{Q^{2}}{2 P \cdot q} \\
\left\langle|\mathscr{M}|^{2}\right\rangle=\left(\frac{e^{2}}{q^{2}}\right)^{2} \mathscr{L}^{\mu v} \mathscr{H}_{\mu v} \\
\mathscr{L}^{\mu v}=q^{2}\left[g^{\mu v}+\frac{2}{q^{2}}\left(k^{\mu} k^{\prime v}+k^{\prime \mu} k^{v}\right)\right]+2 i \lambda \varepsilon^{\mu v \alpha \beta} k_{\alpha} k_{\beta}^{\prime} \\
\mathscr{H}_{\mu v}=J_{\mu}^{\dagger} J_{v} .
\end{gathered}
$$

Here, $\lambda= \pm 1$ indicates the sign of the spin of the polarized beam, $M$ is the mass of the target, and $e$ is the charge of the lepton from the beam. $J^{\mu}$ is the hadronic current; its form is process-dependent and contains information about the hadronic physics.

\subsection{BSA of Pseudoscalar Meson Production}

The most general form of the hadronic current for pseudoscalar meson production is given by

$$
J_{\mathrm{PS}}^{\mu}=i F_{\mathrm{PS}} \varepsilon^{\mu v \alpha \beta} q_{v} \bar{P}_{\alpha} \Delta_{\beta},
$$

where $\bar{P}=P+P^{\prime}$, and $\Delta=P-P^{\prime}$. It should be clear by inspection that this implies $\mathscr{H}_{\mu \nu}=\mathscr{H}_{\nu \mu}$ due to the single form factor. Thus the antisymmetric part of $\mathscr{L}^{\mu v}$, the term with the beam polarization information, does not contribute to the total cross section. Now the BSA is given by

$$
\mathrm{BSA}=\frac{\mathrm{d} \sigma_{\lambda=1}-\mathrm{d} \sigma_{\lambda=-1}}{\mathrm{~d} \sigma_{\lambda=1}+\mathrm{d} \sigma_{\lambda=-1}}
$$

However, because the cross section is independent of beam polarization for pseudoscalar meson production, the numerator vanishes. Due ultimately to the single form factor and lack of BetheHeitler process interference, we thus predict that the BSA for pseudoscalar meson production identically vanishes for all kinematics. This has been experimentally verified by Cao [2] (see Figure 2).

\subsection{BSA of Scalar Meson Production}

While the BSA of pseudoscalar meson production vanishes for all kinematics, this is not the case for scalar meson production. This is due to there being two form factors for the hadronic current:

$$
\begin{aligned}
J_{S}^{\mu} & =\left(S_{q} q_{\alpha}+S_{\bar{P}} \bar{P}_{\alpha}\right) d^{\mu v \alpha \beta} q_{\beta} \Delta_{v} \\
& =F_{1}\left(q^{2} \Delta^{\mu}-q \cdot \Delta q^{\mu}\right)+F_{2}\left[\left(\bar{P} \cdot q+q^{2}\right) \Delta^{\mu}-q \cdot \Delta\left(\bar{P}^{\mu}+q^{\mu}\right)\right],
\end{aligned}
$$

where we have introduced the backbone of the Compton tensor

$$
d^{\mu v \alpha \beta}=g^{\mu v} g^{\alpha \beta}-g^{\mu \alpha} g^{v \beta},
$$

which may be used to construct pieces of "DNA" [3, 4] for virtual Compton scattering as well as meson electroproduction. In the second line of Eq.(2.8), we rewrote the form factors through

$$
\begin{aligned}
& F_{1}=S_{q}-S_{\bar{P}} \\
& F_{2}=S_{\bar{P}} .
\end{aligned}
$$




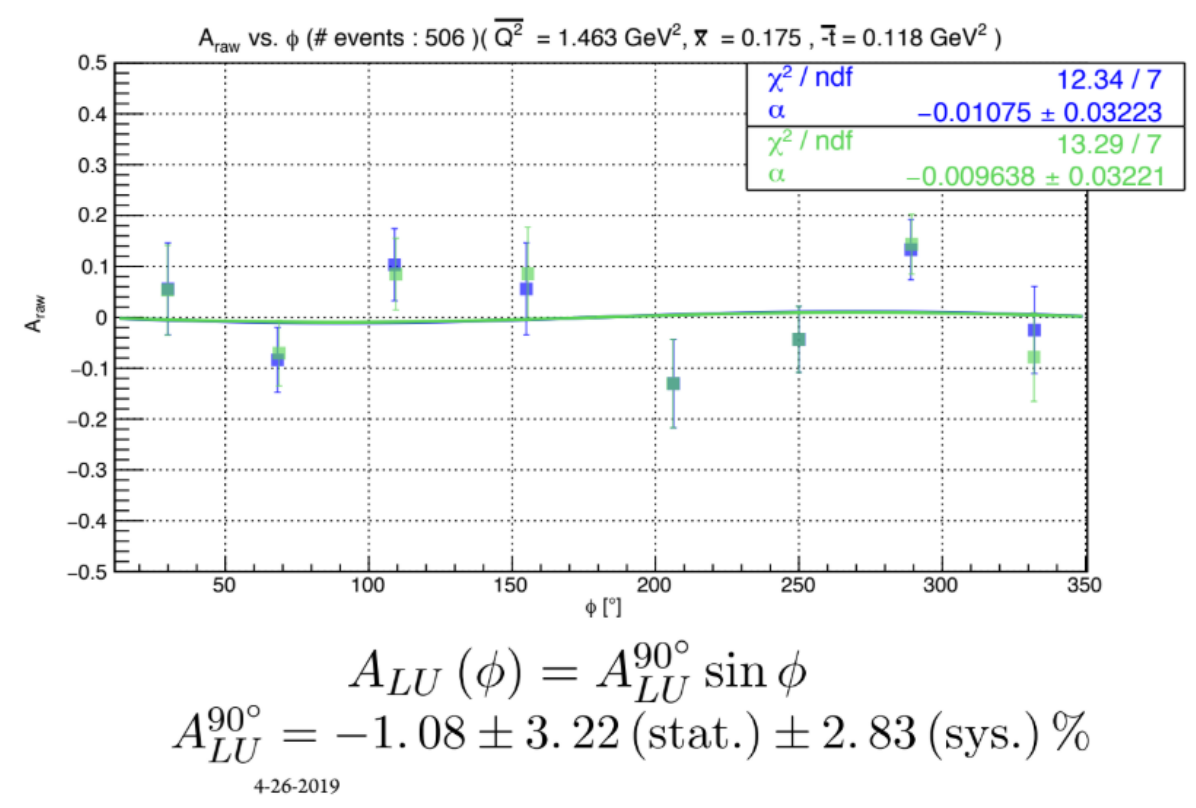

Beam-Spin Asymmetry of Exclusive Coherent Electroproduction of the $\pi 0$ Off $4 \mathrm{He}$

Frank Thanh Cao

University of Connecticut - Storrs, franktcao@gmail.com

Figure 2: Experimental evidence supporting the vanishing BSA for pseudoscalar electroproduction off the scalar target. Figure taken from [2], page 157.

Note that the current is manifestly gauge invariant, and one can see that it reduces to the usual electromagnetic current $J^{\mu} \propto\left(P+P^{\prime}\right)^{\mu}$ for the case of no meson production, i.e., $q^{\prime}=0$. When one evaluates Eq.(2.7) for scalar meson production, it turns out that the BSA is proportional to $F_{1} F_{2}^{*}-F_{1}^{*} F_{2}$. Therefore, if either form factor in Eq.(2.8) has a non-vanishing imaginary part for a given kinematics, we expect the BSA for scalar meson production to be non-vanishing.

\section{Contrast to Leading-Twist GPDs}

The leading-twist GPD formulation [5, 6] provides detailed information of the individual contribution from each and every constituent of the target. However, the GPD formalism relies on the "handbag dominance" and the kinematic region for the handbag dominance is restricted to $|t| \ll Q^{2}$ [7]. Our findings from the general formulation with two independent hadronic form factors for the electroproduction of the scalar $\left(0^{++}\right)$meson may be compared with the GPD formulation discussed in the review [8] which provided the number of leading-twist GPDs for the same process. In particular, one should note that not only the chiral-even operator $\gamma^{+}$but also the chiral-odd operator $\sigma_{\mu}^{+\perp}$ can be effective for spin-zero hadrons, providing the contribution from the two twist-2 GPDs, i.e., the chiral-even GPD $(H)$ and the chiral-odd GPD $\left(H_{T}\right)$, respectively, to the DVMP process of the scalar $\left(0^{++}\right)$meson production. As pointed out in Ref. [8], the GPDs defined by the aligned parton-helicity operators are allowed due to nonzero orbital angular momentum between the initial and final state hadrons. One may check explicitly the helicity flip vs. non-flip 
amplitudes in the quark level including not only the identity coupling to the quark-scalar $\left(0^{++}\right)$meson vertex which singles out the chiral-odd GPD $\left(H_{T}\right)$ but also the derivative coupling with $\gamma_{\mu}$ to the quark-scalar $\left(0^{++}\right)$meson vertex which provides the chiral-even GPD $(H)$ contribution. As the chirality and the helicity coincide in the massless limit, it is rather straightforward to identify the chiral-even vs. chiral-odd contribution from the helicity flip vs. non-flip amplitudes, respectively. According to our evaluation of helicity flip vs. non-flip amplitudes [1], the derivative coupling with $\gamma_{\mu}$ can bring $\sqrt{Q^{2}}$ over the non-derivative identity coupling. While this might naively suggest the $\sqrt{-t} / Q$ suppression of the chiral-odd contribution with respect to the chiral-even contribution, one should note that very little is known on the scalar $\left(0^{++}\right)$meson wave function in the quark-scalar $\left(0^{++}\right)$meson vertex. Overcoming the $\sqrt{-t} / Q$ factor, if the chiral-odd GPD $\left(H_{T}\right)$ contributes as significantly as the chiral-even GPD $(H)$, then the GPD formulation would provide the nonvanishing BSA in DVMP of scalar $\left(0^{++}\right)$meson production off the scalar target as we have discussed with our general formulation in Subsection 2.2. Unless the chiral-odd GPD $\left(H_{T}\right)$ contributes as significantly as the chiral-even GPD $(H)$, a single GPD contribution alone would provide a zero BSA. The measurement of the BSA in the kinematic region $|t| \ll Q^{2}$ may still be analyzed without involving any higher twist GPDs as it has been discussed in Ref. [9]. Thus, the BSA measurement of $f_{0}(980)$ electroproduction off the ${ }^{4} \mathrm{He}$ target would provide a unique opportunity not only to explore the imaginary part of the hadronic amplitude in the general formulation but also to examine the significance of the chiral-odd GPD contribution in the leading-twist GPD formulation.

\section{References}

[1] Chueng-Ryong Ji, Ho-Meoyng Choi, Andrew Lundeen, and Bernard L.G. Bakker, Beam spin asymmetry in the electroproduction of a pseudoscalar meson or a scalar meson off the scalar target, Phys. Rev. D, 99:116008, June 2019.

[2] Frank Thanh Cao, "Beam-Spin Asymmetry of Exclusive Coherent Electroproduction of the $\pi^{0}$ off ${ }^{4} \mathrm{He}^{2}$ (2019), Doctoral Dissertations, University of Connecticut - Storrs.

[3] Chueng-Ryong Ji and Bernard L.G. Bakker, Conceptual issues of GPDs, PoS QCDEV, 2017:038, 2017.

[4] Bernard L.G. Bakker and Chueng-Ryong Ji, The construction of compton tensors in scalar QED, Few Body Syst., 58:8, 2017.

[5] Xiangdong Ji, Gauge-invariant decomposition of nucleon spin, Phys. Rev. Lett., 78:610-613, Jan 1997.

[6] A.V. Radyushkin, Scaling limit of deeply virtual compton scattering, Physics Letters B, 380(3-4):417-425, Jul 1996.

[7] Chueng-Ryong Ji and Bernard L.G. Bakker, Conceptual issues concerning generalized parton distributions, Int. J. Mod. Phys. E, 22:1330002.

[8] A.V. Belitsky and A.V. Radyushkin, Unraveling hadron structure with generalized parton distributions, Physics Reports, 418(1-6):1, Oct 2005.

[9] Hattawy et. al., First exclusive measurement of deeply virtual compton scattering off ${ }^{4}$ He: Toward the 3d tomography of nuclei, Phys. Rev. Lett., 119:202004, Nov 2017. 